\title{
Review: a lack of evidence exists to indicate the most effective treatment for patients who have deliberately harmed themselves
}

\author{
Hawton K, Arensman E, Townsend E, et al. Deliberate self-harm: the efficacy of psychosocial and pharmacological treatments. \\ Cochrane Review, latest version 12 May 1998. In: Cochrane Library. Oxford: Update Software.
}

\section{Question}

In patients who have deliberately harmed themselves, which psychosocial or pharmacological treatments are most effective?

\section{Data sources}

Studies were identified by searching Medline, PsycLIT, EMBASE/Excerpta Medica, and the Cochrane Controlled Trials Register; handsearching 10 journals; reviewing the reference lists of identified articles; and contacting experts.

\section{Study selection}

Studies were selected if they were randomised controlled trials comparing psychosocial or psychopharmacological treatments with standard or less intensive types of aftercare for patients who engaged in any type of deliberately initiated self poisoning or self injury shortly before entering a study.

\section{Data extraction}

Data were extracted on characteristics of the patients, type and details of the intervention, and proportion of patients who repeated suicidal behaviour.

\section{Main results}

20 studies involving 2452 patients with outcome data were included. No difference in the repetition of deliberate self harm existed when problem solving therapy (4 studies), intensive intervention plus outreach (6 studies), or emergency contact card (2 studies) were compared with standard aftercare; inpatient behaviour therapy compared with inpatient insight orientated therapy (1 study); general hospital admission compared with discharge (1 study); antidepressants compared with placebo (2 studies); or long term therapy compared with short term therapy (1 study). A reduced rate of repetition of deliberate self harm was found in the experimental groups when dialectical behaviour therapy was compared with standard aftercare (subgroup analysis from 1 study, 26\% v 60\% $\{\mathrm{p}=0.03\}^{*}$ ), and when flupenthixol was compared with placebo (1 small study, $21 \%$ v $\left.75 \%\{\mathrm{p}=0.003\}^{*}\right)$. A higher rate of subsequent deliberate self harm was found in patients allocated to the same therapist compared with those allocated to a different therapist (1 study, $18 \%$ v 5\%) $\{\mathrm{p}=0.02\} *$ but this finding was probably the result of unequal distribution of patients at risk in the 2 groups.

\section{Conclusion}

There is insufficient evidence to make recommendations on which treatments are the most effective for patients who have recently engaged in deliberate self harm.

*p value calculated from data in article.

Source of funding: NHS Executive Anglia and Oxford Research and Development Programme.

For correspondence: Professor K Hawton, University Department of Psychiatry, Warneford Hospital, Oxford OX3 7JX, UK. Fax +44 (0)1865 793101.

\section{Commentary}

This review by Hawton et al summarises the evidence for psychosocial or pharmacological treatment of individuals after episodes of deliberate self harm. Suicide attempts or deliberate self harm are a frequent cause of contact with mental health services. Although people attempting suicide are frequently encountered, no best practices have been established.

Other reviewers have approached this evidence using different definitions and conceptual models. Using broader inclusion criteria our own review of psychosocial interventions included the study by Rudd $e t a l$, which was excluded by Hawton et al because the sample included individuals with suicidal ideation without attempts. ${ }^{12}$ Their groupings of studies seem to be less conceptually based than those used by Van der Sande $e t a l .^{3}$ Their review and meta-analysis grouped together interventions using a cognitive behavioural rationale and reported a summary relative risk ratio for these stud- ies that was statistically significant. Other work has been published since the review by Hawton $e t$ al. A recent trial showed that for individuals with $<5$ previous attempts, a statistically significant reduction in recurrent suicide attempts occurred in the paroxetine arm compared with the placebo arm. ${ }^{4}$

We have cautioned that although statistical significance has been difficult to show, several trials have reported reductions which may have clinical significance. The literature clearly indicates that treatment in the post-discharge period, by assertive follow up of individuals with recent self harm, is warranted.

\section{Paul S Links, MD, FRCP(C) St Michael's Hospital Toronto, Ontario, Canada}

1 Rudd MD, Rajab MH, Orman DT, et al. Effectiveness of an outpatient intervention targeting suicidal young adults: preliminary 90.
2 Rhodes AE, Links PS. Suicide and suicidal behaviors: implications for mental health services. Can J Psychiatry 1998;43:785-91.

3 van der Sande R, Buskens E, Allart E, et al. Psychosocial intervention following suicide attempt: a systematic review of treatment interventions. Acta Psychiatr Scand 1997:96: 43-50.

4 Verkes RJ, Van der Mast RC, Hengeveld MW, et al. Reduction by paroxetine of suicidal behavior in patients with repeated suicide attempts but not major depression. Am J Psychiatry 1998;155:543-7.

\section{Authors' response}

Dr Links indicates that our grouping of studies was "less conceptually based than that used by Van der Sande et al' and particularly highlights the category of cognitive behavioural treatment in which these authors included dialectical behaviour therapy (DBT). It is inappropriate to include Linehan's study of DBT with studies of brief (up to 10 sessions) problem solving therapy as the former was a 1 year intensive programme of individual and group therapy. 